\title{
CO-REGISTRATION OF CARDIAC MAGNETIC RESONANCE (CMR) IMAGES FROM PRETERM INFANTS: PILOT WORK IN CREATING A NOVEL NEONATAL CARDIAC ATLAS
}

\author{
D. Cox $^{1}$, W. Shi ${ }^{2}$, A.M. Groves ${ }^{1}$, A.N. Price ${ }^{1}$, G. Durighel ${ }^{1}$, K.M. Broadhouse ${ }^{1}$, A.E. Finnemore ${ }^{1}$, A.D. \\ Edwards $^{1}$, D. Rueckert ${ }^{2}$ \\ ${ }^{I}$ Centre for the Developing Brain, Imperial College London, MRC Centre for Clinical Sciences, ${ }^{2}$ Biomedical \\ Image Analysis Group, Department of Computing, Imperial College, London, UK
}

Background and aims: Preterm circulatory failure is a major cause of infant morbidity but remains poorly understood.

Adult cardiac magnetic resonance (CMR) atlases aid detection of ventricular anatomical and wall motion abnormalities. We hypothesize that constructing a preterm CMR atlas will enable identification of similar pathologies whilst providing robust functional biomarkers needed to guide future preterm inotrope trials.

Cardiac atlas creation requires alignment of images obtained throughout the cardiac cycle to a common reference space (co-registration); the degree of warping necessary to align individual images measures regional anatomical variability.

This study examines the feasibility of co-registering neonatal CMR images.

Methods: 5 free-breathing, unsedated preterm infants (corrected gestational age 31+4-32+6weeks, birthweight $1.7-1.9 \mathrm{~kg}$ ), underwent CMR in a $3.0 \mathrm{~T}$ dedicated neonatal scanner.

4-chamber, ventricular long axis and short axis stack views were acquired using steady state free procession sequences with resolution $1.5 \times 1.5 \times 5 \mathrm{~mm}, 32$ phases/cardiac cycle, $2 \mathrm{NSA}$. $2.5 \mathrm{~mm}$ slice overlap provided 10 short axis slices from ventricular apex to base.

Co-registration was performed using non-rigid computational algorithms that automatically identified: cardiac apex, right ventricular insertion points, most lateral right and left ventricular free wall points, basal short axis slice centre-point, and tissue contrast differences.

Results: Co-registration of the CMR images is shown.

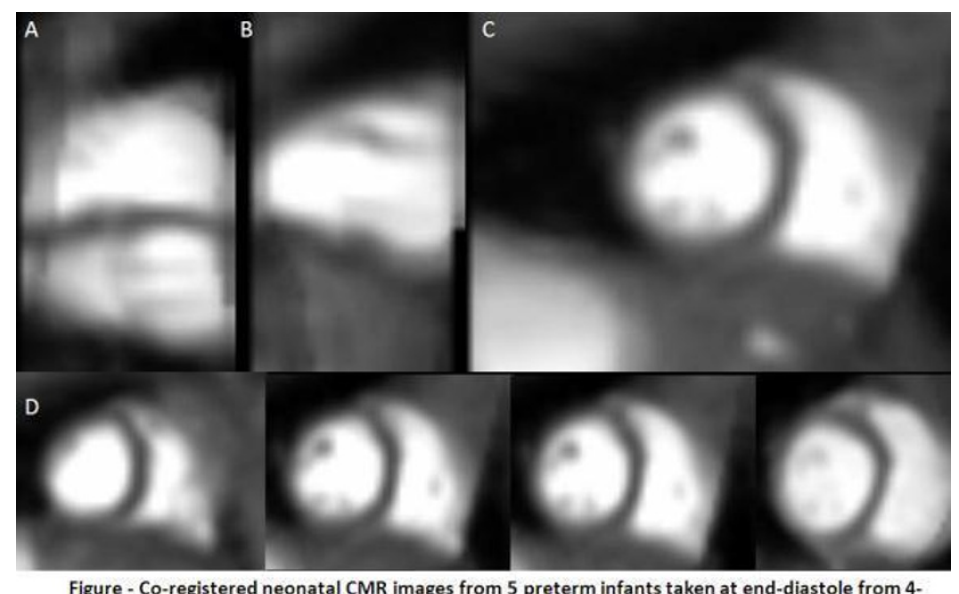

Figure - Co-registered neonatal CMR images from 5 preterm infants taken at end-diastole from 4[Co-registered ventricular atlas of 5 preterm scans]

Conclusions: This work demonstrates successful preterm CMR image co-registration - the critical first step to producing a functional neonatal CMR atlas. 